\title{
Diagnosis of quantum criticality by nuclear spin-echo decay method
}

\author{
S. Kambe ${ }^{*, 1}$, H. Sakai ${ }^{1}$, Y. Tokunaga ${ }^{1}$, H. Chudo ${ }^{1}$, and R. E. Walstedt ${ }^{2}$ \\ ${ }^{1}$ Advanced Science Research Center, Japan Atomic Energy Agency, Tokai-mura, Ibaraki 319-1195, Japan \\ ${ }^{2}$ Physics Department, The University of Michigan, Ann Arbor, MI 48109, USA
}

Received 10 July 2009, revised 1 September 2009, accepted 7 September 2009

Published online 16 February 2010

PACS 74.25.Nf, 74.40.+k

*Corresponding author: e-mail kambe.shinsaku@jaea.go.jp, Phone: +81-29-2843525, Fax: +81-29-2825927

The Gaussian spin-spin relaxation time $T_{2 \mathrm{G}}$, as determined by nuclear magnetic resonance (NMR) spin-echo decay measurements, is found to be useful for diagnosis of quantum critical behaviour in heavy-fermion systems. Combining $T_{2 \mathrm{G}}$ with the spin-lattice relaxation time $T_{1}$, the exponent $\phi$ of the quantity
$T_{1} T / T_{2 G}^{2} \sim T^{\phi}$ is predicted to be sensitive to the type of quantum criticality in the system concerned. In fact, in the heavy-fermion system $\mathrm{USn}_{3}$ near the quantum critical point, $T_{1} T / T_{2 G}^{2}$ is found to be constant, as expected for a 3D-SDW magnetic instability.
1 Introduction Investigations of quantum critical point $(\mathrm{QCP})$ behaviour have been triggered by the pioneering work of von Löhneysen's group on the heavy-fermion system $\mathrm{CeCu}_{1-x} \mathrm{Au}_{x}$ in the 1990s [1], although the particular case of a quantum phase transition for spin density wave (SDW) instability had already been predicted by Hertz in the 1970s [2]. As pointed out by von Löhneysen, heavy-fermion systems are good candidates for investigating QCP behaviour, since a characteristic lowest energy excitation can be tuned to zero easily owing to the competition between the RKKY interaction and the Kondo effect.

Stimulated by this idea, workers have studied quantum critical behaviour in many heavy-fermion systems during the last decade [3]. In these studies, we recognize that many features can relate to the QCP, e.g. local criticality (Kondo break down) [4], valence transitions [5] and Lifshitz (topological) transitions [6]. Since any low-energy excitations can become significant at a QCP, interactions that are negligible for systems far from a QCP are no longer negligible near the QCP. As the system approaches a QCP, relevant interactions may tend to become more complex, leading to several crossover behaviours.

In this context, we have also learned that it is quite difficult to realize ideal quantum critical behaviour which can be described with a simple model having only one relevant interaction, although such complexity may induce a novel phase. In any case, it is necessary to categorize the
QCP in a way which takes account of the types of relevant interactions.

In this paper, we present a nuclear magnetic resonance (NMR) spin-echo decay method, which we employ as a tool for categorizing a QCP. It is well-known that this method has been quite useful to clarify the magnetic properties of high $T_{c}$ cuprates [7]. As the first application of the method to heavy fermion systems, spin-echo decay results near the QCP in $\mathrm{USn}_{3}$ are presented.

2 Spin-lattice and spin-spin relaxation times In NMR, two significant relaxation processes, spin-lattice relaxation and spin-spin relaxation, are defined. The spinlattice (longitudinal) relaxation time $\left(T_{1}\right)$ represents the characteristic time for recovery of longitudinal (i.e. along the applied magnetic field $H$ ) nuclear magnetization through energy dissipation. This process is related to the dynamical susceptibility $\operatorname{Im} \chi(q, \omega)$ of a $D$-dimensional electronic system [8],

$$
\frac{1}{T_{1} T} \sim \int \frac{\operatorname{Im} \chi\left(q, \omega_{n}\right)}{\omega_{n}} d^{D} q
$$

where $\omega_{n}$ is the NMR measurement frequency. In contrast, the spin-spin (transverse) relaxation time $\left(T_{2}\right)$ represents the characteristic time for decoherence of transverse nuclear magnetization (i.e. perpendicular to $H$ ) without energy 
dissipation. Usually, $T_{2}$ is determined by means of nuclear spin-echo decay measurements. The dependence of the nuclear spin-echo intensity $M$ on the echo delay time $2 \tau$ is expressed [9],

$$
M(2 \tau)=M(0) \exp \left(-\frac{2 \tau}{T_{2 \mathrm{~L}}}\right) \exp \left(-\frac{(2 \tau)^{2}}{2 T_{2 \mathrm{G}}^{2}}\right),
$$

where $\tau$ is the time interval between first and second radiofrequency (RF) pulses, and the two exponential factors are the Lorentzian and Gaussian components, respectively. In Fig. 1, spin-echo formation by RF pulses and a purely Gaussian relaxation decay curve (the case for $T_{2 \mathrm{~L}}=\infty$ in Eq. (2)) are presented.

The Lorentzian decay rate $1 / T_{2 \mathrm{~L}}$ is related to $1 / T_{1}$ as $1 / T_{2 \mathrm{~L}}=1 / T_{1}$ for the isotropic case. We are interested in the Gaussian rate $1 / T_{2 \mathrm{G}}$, which is related to the static susceptibility $\operatorname{Re} \chi(q, 0)$ of the electronic system when the nuclear indirect coupling is strong [10,11],

$$
\left(\frac{1}{T_{2 \mathrm{G}}}\right)^{2} \sim \int \operatorname{Re} \chi(q, 0)^{2} d^{D} q
$$

In this report, we call attention to the exponent $\phi$ defined by $T_{1} T / T_{2 \mathrm{G}}^{2} \sim T^{\phi}$. Thus, the hyperfine coupling constant $A(q)$ is not treated explicitly in Eqs. (1) and (3), since $\phi$ is approximately independent of $A(q)$.

3 Several magnetic cases In order to distinguish the origin of quantum criticality using $T_{1}$ and $T_{2 \mathrm{G}}$, the $T$ dependences of $T_{1}$ and $T_{2 \mathrm{G}}$ are described for three different cases, viz. (i) a 3D SDW instability, (ii) the local-moment state and (iii) a 2D locally critical instability. In particular, this is the first quantitative estimate for the last case.

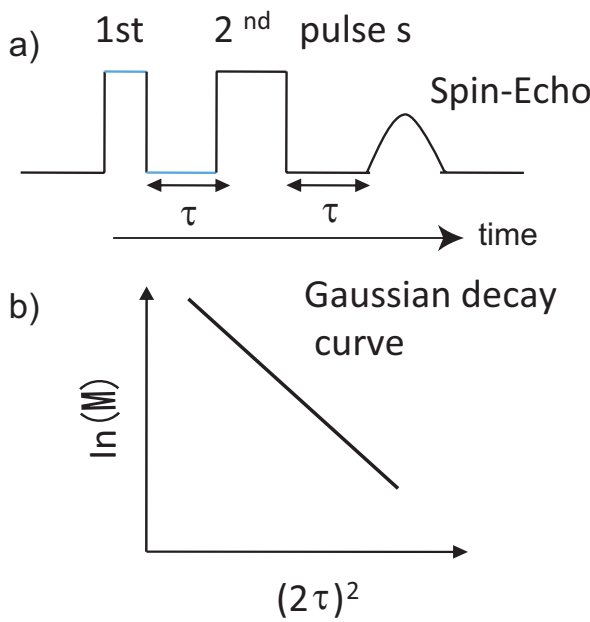

Figure 1 (online colour at: www.pss-b.com) (a) Pulsed NMR spinecho decay is depicted. As the time interval $\tau$ between the two RF pulses increases, the spin-echo intensity decays due to spin-spin relaxation processes. (b) Spin-echo intensity $M$ as function of $(2 \tau)^{2}$ for the purely Gaussian case $\left(T_{2 \mathrm{~L}}=\infty\right.$ in Eq. (2)). The slope of the straight line corresponds to $-1 / 2 T_{2 \mathrm{G}}^{2}$.
Although the $T$-dependences of $T_{1}$ and $T_{2 \mathrm{G}}$ can differ individually for each case, the quantity $T_{1} T / T_{2 \mathrm{G}}^{2}$ is found to be a better determinant of the origin, since this quantity is independent of detailed electronic structure, in contrast with values of the time constants $T_{1}$ and $T_{2 \mathrm{G}}$ per se.

3.1 The SDW instability case $(\boldsymbol{D}=\mathbf{3})$ Near the 3D itinerant antiferromagnetic SDW instability, the dynamical scaling relation for magnetic susceptibility may be applied [12],

$$
\chi(q, \omega)=\xi^{2-\eta} g\left(\xi q, \xi^{z} \omega\right),
$$

where $\xi$ is the coherence length, $g$ is a scaling function and the critical exponents are $z=2$ and $\eta=0$ for a 3D antiferromagnetic SDW instability which is considered to be in overdamped regime. Based on Eqs. (1), (3) and (4), 1/T 1 and $\left(1 / T_{2 \mathrm{G}}\right)^{2}$ are expressed as,

$$
\frac{1}{T_{1} T} \sim \xi^{z-1-\eta}=\xi, \quad\left(\frac{1}{T_{2 \mathrm{G}}}\right)^{2} \sim \xi^{1-2 \eta}=\xi
$$

Thus,

$$
\frac{T_{1} T}{T_{2 \mathrm{G}}^{2}} \sim \text { constant. }
$$

It should be noted that the relation in Eq. (6) is found to hold in 2D systems such as the optimally-doped high- $T_{\mathrm{c}}$ cuprate $\mathrm{YBa}_{2} \mathrm{Cu}_{3} \mathrm{O}_{7}$ in the overdamped regime (see below).

As a characteristic of a QCP deriving from a 3D antiferromagnetic SDW instability, $\xi$ diverges as $\xi(T) \sim T^{-3 / 4}$, in contrast to $\xi(T)^{-2} \sim \xi(0)^{-2}+A T^{2}$ in the Fermi-liquid regime [13].

3.2 Localized moment case in the high- $T$ limit $(\boldsymbol{D}=3)$ At high temperatures, the heavy-fermion state is effectively transformed into a localized-moment state due to the disappearance of coherent propagation effects. In the localized-moment state, $T_{1} \sim$ const and $T_{2 \mathrm{G}} \sim$ const [8], leading to

$$
\frac{T_{1} T}{T_{2 \mathrm{G}}^{2}} \sim T .
$$

3.3 The antiferromagnetic locally critical case $(\boldsymbol{D}=\mathbf{2})$ In the antiferromagnetic, local QCP model for quasi-2D systems, the magnetic susceptibility near the QCP may be expressed as [4],

$$
\begin{aligned}
& \frac{1}{\chi(q-Q, \omega)} \\
& =c\left(\frac{q-Q}{q_{B}}\right)^{2}+(2 \pi L T)^{\alpha} \exp \left[\alpha \psi\left(\frac{1}{2}-\frac{i \omega}{2 \pi T}\right)\right],
\end{aligned}
$$




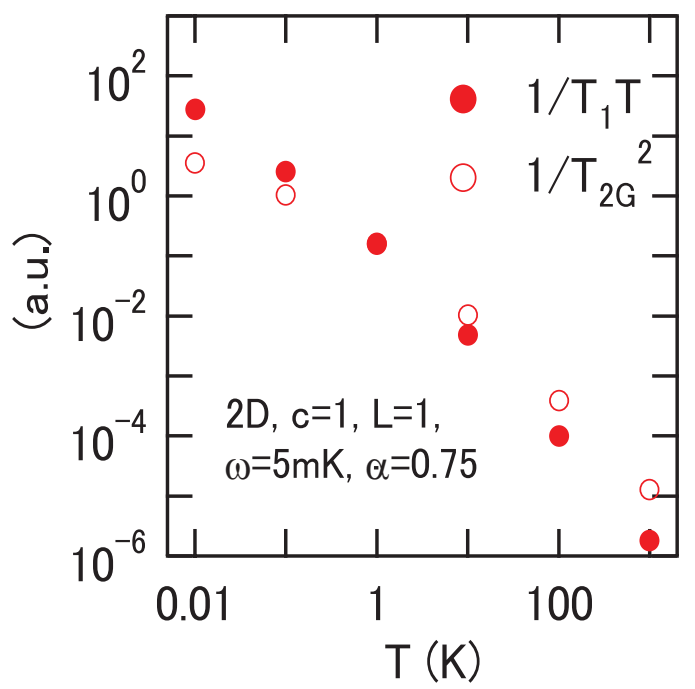

Figure 2 (online colour at: www.pss-b.com) Calculated $T$-dependence of $1 / T_{1} T$ and $1 / T_{2 \mathrm{G}}^{2}$ for the locally critical case based on Eq. 8 .

where $Q$ is the ordering wave vector, $c$ is the dispersion constant, $\boldsymbol{q}_{\mathrm{B}}$ is the zone-boundary wavevector, $L \equiv \Lambda_{0}^{1 / \alpha} / \Lambda$ is a constant of order of unity, $\psi$ is the digamma function and $\Lambda \sim$ the Kondo temperature (see Ref. [4] for the definitions of $\Lambda$ and $\Lambda_{0}$ ). Anomalous, locally QCP behaviour appears when the exponent $\alpha \neq 1$. For example, $\alpha \sim 0.75$ has been found [14] for the case of $\mathrm{CeCu}_{0.9} \mathrm{Au}_{0.1}$. Based on this model, the spin-lattice and spin-spin relaxation times can be calculated through $2 \mathrm{D} q$-integration of Eq. 8. For example, the $T$-dependences of $1 / T_{1} T,\left(1 / T_{2 \mathrm{G}}\right)^{2}$ and $T_{1} T / T_{2 \mathrm{G}}^{2}$ in arbitrary units for $c=L=1[15], \alpha=0.75$ and $\omega_{n}=5 \mathrm{mK}$ are presented in Fig. 2. In contrast with the localized-moment case, $1 / T_{1}$ and $\left(1 / T_{2 \mathrm{G}}\right)^{2}$ are $T$-dependent. At temperatures $T \gg \omega_{n}, T_{1} T / T_{2 \mathrm{G}}^{2}$ is expressed approximately (solid lines in Fig. 3) as

$$
\frac{T_{1} T}{T_{2 \mathrm{G}}^{2}} \sim T^{\phi} \sim T^{1-\alpha} .
$$

This value of exponent $\phi$ depends on $\alpha$ as $\phi \sim 1-\alpha$, as shown in Fig. 4, and is rather insensitive to the values of $L$ and $c$ around the present case. As expected, the SDW relation $T_{1} T / T_{2 \mathrm{G}}^{2} \sim$ const is recovered for the case of $\alpha=1$. Further, the $\alpha=0$ case corresponds to the localized-moment case in mathematical form. Thus, the locally critical case looks to interpolate between these two cases.

At low temperatures comparable to $\omega_{n}$, the $T$-dependence of $T_{1} T / T_{2 \mathrm{G}}^{2}$ becomes non-universal, suggesting that the NMR measurement frequency $\omega_{n} \ll T$ is required to determine the universal exponent $\phi$.

In Table 1, the $T$-dependence of $T_{1} T / T_{2 \mathrm{G}}^{2}$ is summarized for three cases, indicating that the quantity $T_{1} T / T_{2 \mathrm{G}}^{2}$ is useful for determining the type of magnetic quantum criticality. It should be noted that different exponents $\phi$ are found for ferromagnetic cases, which will be presented elsewhere.

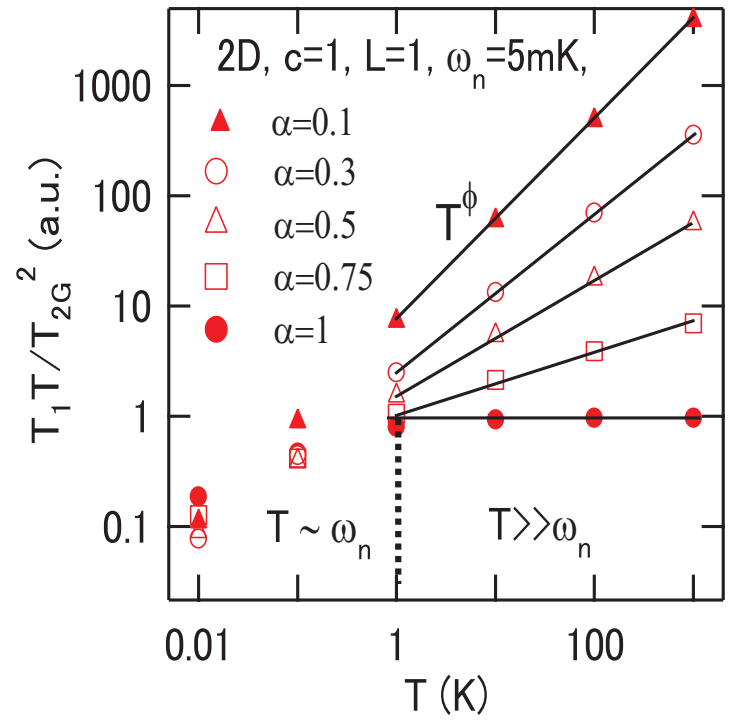

Figure 3 (online colour at: www.pss-b.com) $T$-dependence of $T_{1} T / T_{2 \mathrm{G}}^{2}$ for various values of $\alpha$. The solid lines are obtained from least-squares fittings of $\phi$ for data above $1 \mathrm{~K}\left(T \gg \omega_{n}\right)$ using the equation $T_{1} T / T_{2 \mathrm{G}}^{2}=T^{\phi}$.

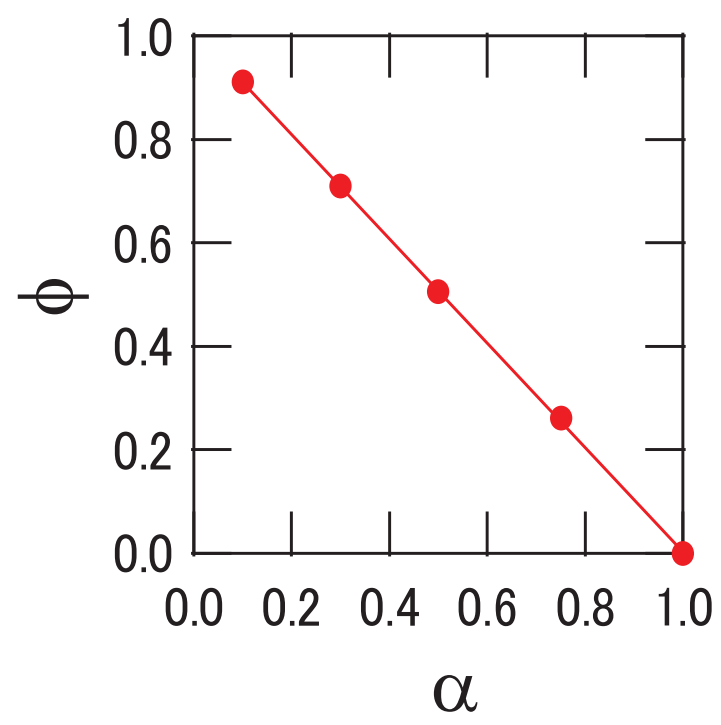

Figure 4 (online colour at: www.pss-b.com) The $\alpha$-dependence of $\phi$ estimated from the slopes of solid lines in Fig. 3. The solid line represents the relation $\phi=1-\alpha$.

Table 1 The $T$-dependence of $T_{1} T / T_{2 \mathrm{G}}^{2}$ in three different cases: (i) 3D-antiferromagnetic SDW instability; (ii) localized-moment state at high $T$; (iii) 2D-locally quantum critical instability $(1 \geq \alpha \geq 0)$.

\begin{tabular}{llll}
\hline & (i) $3 \mathrm{D}-\mathrm{SDW}$ & (ii) localized moments & (iii) 2D-local \\
\hline$T_{1} T / T_{2 \mathrm{G}}^{2}$ & $\sim \mathrm{const}$ & $\sim T$ & $\sim T^{1-\alpha}$ \\
\hline
\end{tabular}




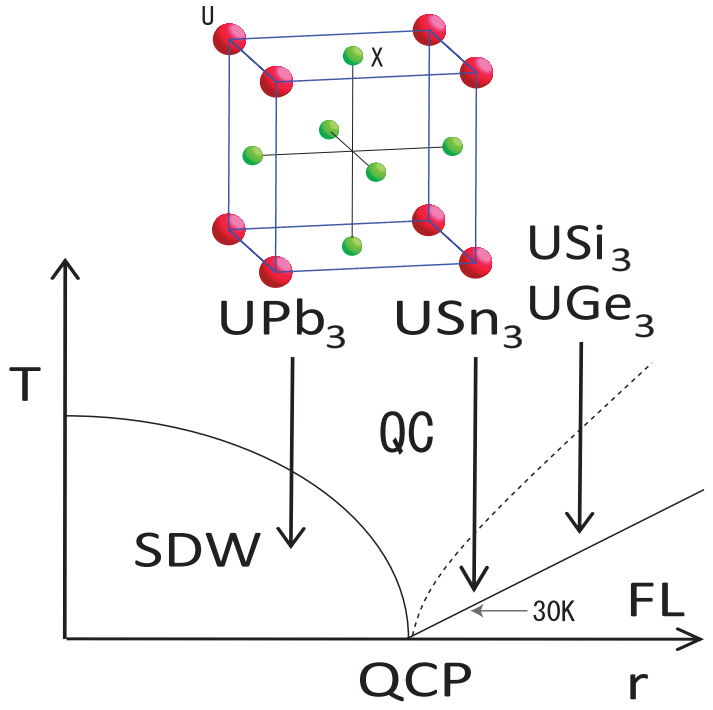

Figure 5 (online colour at: www.pss-b.com) Phase diagram for $\mathrm{UX}_{3}$ ( $X$ : IVB group) compounds. QC: quantum critical regime; FL: Fermi-liquid regime. The dashed line represents a crossover from QC to an 'intermediate' regime. The variable $r$ represents a control parameter for magnetic instability. $\mathrm{USn}_{3}$ is considered to be located just near the QCP. The cubic (FCC) crystal structure of $\mathrm{UX}_{3}$ is presented above the phase diagram.

4 An example of the SDW case: USn ${ }_{3}$ The heavyfermion compound $\mathrm{USn}_{3}$ has a large Sommerfeld specific heat constant $\gamma=170 \mathrm{~mJ} / \mathrm{mol} \mathrm{K}^{2}$ with a paramagnetic ground state [16]. Among $\mathrm{UX}_{3}$ ( $X$ : IVB group) compounds [17], $\mathrm{USn}_{3}$ is located near a QCP as shown in Fig. 5. As measured from the QCP point based on the spin-fluctuation model, $y_{0} \equiv 1 /\left(2 T_{\mathrm{A}} \chi_{Q}(0 \mathrm{~K})\right) \sim 0.2[18]$ is rather small ( $y_{0}=0$ at the QCP, here $T_{\mathrm{A}}$ is the characteristic spinfluctuation temperature [19]). Actually, an enhancement of antiferromagnetic correlations is observed in inelastic neutron scattering measurements [20]. The heavy-fermion state appears below the coherence temperature $T^{*}$, which has been estimated as $\sim 30 \mathrm{~K}$ [16]. The $T$-dependences of $T_{1}$ and $T_{2 \mathrm{G}}$ have been measured at the $\mathrm{Sn}$ site using the usual spinecho method at $\omega_{n}=119 \mathrm{MHz} \sim 6 \mathrm{mK}$. Experimental details have been described in previous reports [18, 21].

Figure 6 a shows the $T$-dependence of $T_{1} T / T_{2 \mathrm{G}}^{2}$ in $\mathrm{USn}_{3}$. Below $T^{*} \sim 30 \mathrm{~K}$ in the heavy-fermion state, the relation $T_{1} T / T_{2 \mathrm{G}}^{2} \sim$ const is found, indicating that the ground state of $\mathrm{USn}_{3}$ is the Fermi-liquid state near to the SDW instability as shown in Fig. 5. At high temperatures, $T_{1} T / T_{2 \mathrm{G}}^{2}$ becomes $T$ dependent, $T_{1} T / T_{2 \mathrm{G}}^{2} \sim T^{0.7}$. The localized state does not appear below $300 \mathrm{~K}$, since $\phi<1$. This looks like the locally critical case with $\alpha \sim 0.3$; however, since $\mathrm{USn}_{3}$ is a completely isotropic, 3D system, this is not the case. Since $T_{1} T / T_{2 \mathrm{G}}^{2}$ increases with increase in $T, \operatorname{Im} \chi\left(q, \omega_{n}\right) / \omega_{n}$ decreases more rapidly than that expected for $\operatorname{Re} \chi(q, 0)$ from Eq. (4). We believe that $\phi \sim 0.7$ is a characteristic of the heavy-fermion formation process, which breaks the dynamical scaling law at the low-energy of $\omega_{n}$ [21]. The a)

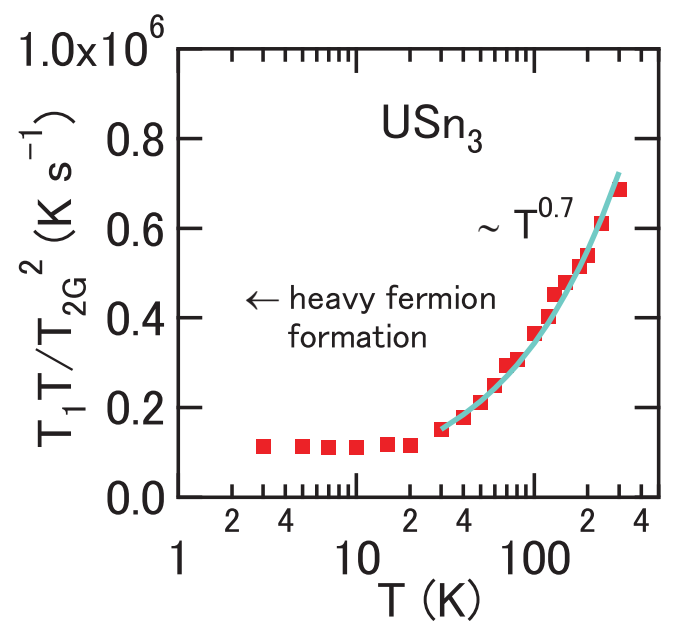

b)

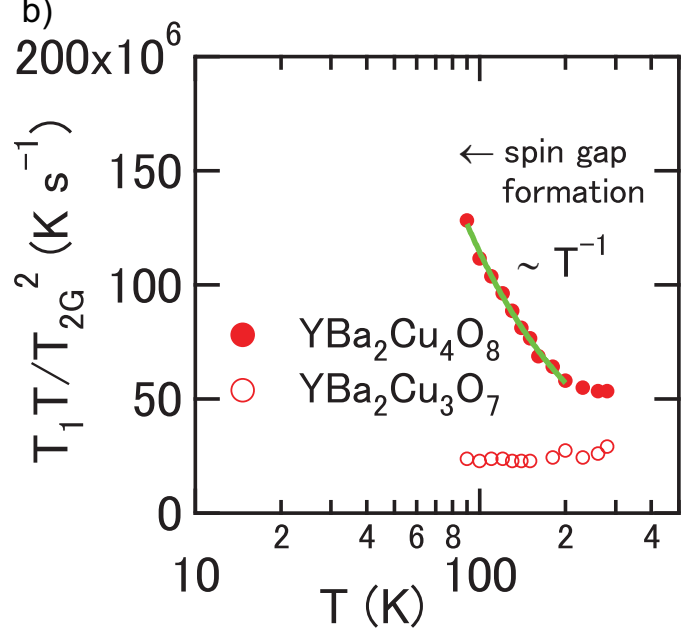

Figure 6 (online colour at: www.pss-b.com) The $T$-dependence of $T_{1} T / T_{2 \mathrm{G}}^{2}$ in $\mathrm{USn}_{3}, \mathrm{YBa}_{2} \mathrm{Cu}_{4} \mathrm{O}_{8}$ and $\mathrm{YBa}_{2} \mathrm{Cu}_{3} \mathrm{O}_{7}$. (a) Heavy-fermion system $\mathrm{USn}_{3}$. Solid line represents $T_{1} T / T_{2 \mathrm{G}}^{2} \propto T^{0.7}$. (b) High $\mathrm{T}_{\mathrm{c}}$ cuprates $\mathrm{YBa}_{2} \mathrm{Cu}_{4} \mathrm{O}_{8}$ (spin-gap case) and $\mathrm{YBa}_{2} \mathrm{Cu}_{3} \mathrm{O}_{7}$ (optimallydoped case) from Ref. [22]. Solid line represents $T_{1} T / T_{2 \mathrm{G}}^{2} \propto T^{-1}$. In the heavy-fermion state of $\mathrm{USn}_{3}$ below $30 \mathrm{~K}, T_{1} T / T_{2 \mathrm{G}}^{2} \sim$ const behaviour is observed. In the heavy-fermion formation process of $\mathrm{USn}_{3}, T_{1} T / T_{2 \mathrm{G}}^{2}$ decreases with decrease in $T$. In contrast, in the spingap formation process, $T_{1} T / T_{2 \mathrm{G}}^{2}$ increases with decrease in $T$. In the $2 \mathrm{D}$ overdamped regime, e.g. $\mathrm{YBa}_{2} \mathrm{Cu}_{3} \mathrm{O}_{7}, T_{1} T / T_{2 \mathrm{G}}^{2} \sim$ const behaviour is observed.

observed small $\phi$ may be a characteristic of U-based heavyfermion compounds owing to the strongly itinerant nature of $5 \mathrm{f}$-electrons. In more localized $4 \mathrm{f}-\mathrm{Ce}$ heavy-fermion compounds, one expects $\phi$ to be close to unity. This needs to be confirmed.

It is suggestive to compare this result with the $T$ dependence of $T_{1} / T_{2 \mathrm{G}}^{2}$ in spin-gap and optimally-doped 2D high- $T_{\mathrm{c}}$ cuprates [22] (Fig. 6b). In an optimally-doped compound which is considered to be in the overdamped regime, $T_{1} T / T_{2 \mathrm{G}}^{2}$ is constant. In contrast, $T_{1} T / T_{2 \mathrm{G}}^{2}$ increases with decrease in $T$ in spin-gap compounds, whose variation is 
in the opposite sense compared with the heavy-fermion case. In the spin-gap regime, low-energy spin excitations decrease due to the gap opening. In contrast, heavy-fermion formation corresponds to an increase of low-energy magnetic excitations with decrease in $T$, which may be consistent with the development of a Kondo-coherence peak.

In the spin-gap regime, $T_{1} T / T_{2 \mathrm{G}}^{2}$ can be approximately expressed as $T_{1} T / T_{2 \mathrm{G}}^{2} \sim T^{\phi}$ with $\phi \sim-1$ (Fig. 6b). Although the physical meaning of the exponent $\phi$ for spingap and heavy-fermion formation is still unclear, it would be useful if we could treat heavy-fermion formation and spingap behaviour in a unified framework which reproduces the $T$-dependence of $T_{1} T / T_{2 \mathrm{G}}^{2}$.

5 Conclusions In an effort to distinguish the type of magnetic quantum criticality, the $T$-dependences of $T_{1}, T_{2 \mathrm{G}}$ and especially $T_{1} T / T_{2 \mathrm{G}}^{2}$ are found to be useful. The exponent $\phi$ of $T_{1} T / T_{2 \mathrm{G}}^{2} \sim T^{\phi}$ is suggested to be a good measure of magnetic quantum criticality. In the heavy-fermion compound $\mathrm{USn}_{3}, T_{1} T / T_{2 \mathrm{G}}^{2} \sim$ const, i.e. $\phi \sim 0$ at low temperatures, indicating that $\mathrm{USn}_{3}$ is located near to a SDW instability. On the other hand, above the coherence temperature $\sim 30 \mathrm{~K}, \phi \sim 0.7$ owing to heavy-fermion formation.

It would be informative to measure the $T$-dependence of $T_{1} T / T_{2 \mathrm{G}}^{2}$ in many different cases to diagnose quantum criticality. However, a solid solution system such as $\mathrm{CeCu}_{1-x} \mathrm{Au}_{x}$ ) may not be good candidate, because $T_{2 \mathrm{G}}$ is particularly sensitive to disorder-induced local inhomogeneities. Thus, we need to seek out stoichiometric compounds which would be better candidates. This remains a challenge for the future.

Finally, concerning the quantum criticality of different origins such as valence transitions, the $T$-dependence of $T_{1} T / T_{2 \mathrm{G}}^{2}$ can vary considerably from the present discussion, although to date no theoretical calculations have been reported.

Acknowledgements We thank Y. Haga and T. D. Matsuda for preparing high quality samples, Y. Itoh, S. Raymond, H. Yasuoka, G. H. Lander and J. Flouquet for stimulating discussions. This work was supported by a Grant-in-Aid for Scientific Research on Innovative Areas 'Heavy Electrons' of The Ministry of Education, Culture, Sports, Science and Technology, Japan.

\section{References}

[1] H. v. Löhneysen, J. Phys.: Condens. Matter 8, 9689 (1996).

[2] J. A. Hertz, Phys. Rev. 24, 1165 (1976).

[3] H. v. Löhneysen, A. Rosch, K. Vojta, and P. Wölfle, Rev. Mod. Phys. 79, 1015 (2007).

[4] Q. Si, S. Rabello, K. Ingersent, and J. L. Smith, Nature 413, 804 (2001).

[5] S. Watanabe, A. Tsuruta, K. Miyake, and J. Flouquet, Phys. Rev. Lett. 100, 236401 (2008).

[6] P. M. C. Rourke, A. McCollam, G. Lapertot, G. Knebel, J. Flouquet, and S. R. Julian, Phys. Rev. Lett. 101, 237205 (2008).

[7] R. E. Walstedt, The NMR Probe of High-T ${ }_{c}$ Materials (Springer, Berlin, 2008).

[8] T. Moriya, Prog. Theor. Phys. 16, 641 (1956).

[9] N. J. Curro, T. Imai, C. P. Slichter, and B. Dabrowski, Phys. Rev. B 56, 877 (1997).

[10] C. H. Pennington and C. P. Slichter, Phys. Rev. Lett. 66, 381 (1991).

[11] R. E. Walstedt and S.-W. Cheong, Phys. Rev. B 51, 3163 (1995).

[12] For example, P. M. Chaikin and T. C. Lubensky, Principles of Condensed Matter Physics (Cambridge University Press, Cambridge, 1995).

[13] S. Sachdev, Quantum Phase Transitions (Cambridge University Press, Cambridge, 1999).

[14] A. Schröder, G. Aeppll, R. Coldea, M. Adams, O. Stockert, H. v. Löhneysen, E. Bucher, R. Ramasashvill, and P. Coleman, Nature 407, 351 (2000).

[15] Here the dimension of $c$ and $L$ is $[e]$ and $\left[e^{\frac{1}{\alpha}-1}\right]$, respectively, where $e$ is a certain energy unit.

[16] M. R. Norman, S. D. Bader, and H. A. Kierstead, Phys. Rev. B 33, 8035 (1986).

[17] D. Kaczorowski, J. Phys. Soc. Jpn. 75, Suppl. 68 (2005).

[18] S. Kambe, H. Sakai, Y. Tokunaga, T. D. Matsuda, Y. Haga, H. Chudo, and R. E. Walstedt, Phys. Rev. B 77, 134418 (2008).

[19] T. Moriya and T. Takimoto, J. Phys. Soc. Jpn. 64, 960 (1995).

[20] M. Loewenhaupt and C.-K. Loong, Phys. Rev. B 41, 9294 (1990).

[21] S. Kambe, H. Sakai, Y. Tokunaga, T. D. Matsuda, Y. Haga, H. Chudo, and R. E. Walstedt, Phys. Rev. Lett. 102, 037208 (2009).

[22] Y. Itoh and H. Yasuoka, J. Phys. Soc. Jpn. 63, 2518 (1994). 